\title{
Global Structure of Positive Solutions of a Discrete Problem with Sign-Changing Weight
}

\author{
Ruyun Ma, ${ }^{1}$ Chenghua Gao, ${ }^{1}$ Xiaoling Han, ${ }^{1}$ \\ and Xiaoqiang Chen ${ }^{2}$ \\ ${ }^{1}$ Department of Mathematics, Northwest Normal University, Lanzhou 730070, China \\ ${ }^{2}$ School of Automation and Electrical Engineering, Lanzhou Jiaotong University, Lanzhou 730070, China \\ Correspondence should be addressed to Ruyun Ma, ruyun_ma@126.com
}

Received 27 April 2011; Accepted 28 June 2011

Academic Editor: Zengji Du

Copyright (C) 2011 Ruyun Ma et al. This is an open access article distributed under the Creative Commons Attribution License, which permits unrestricted use, distribution, and reproduction in any medium, provided the original work is properly cited.

Let $T>5$ be an integer, $\mathbb{T}=\{1,2, \ldots, T\}$. We are concerned with the global structure of positive solutions set of the discrete second-order boundary value problems $\Delta^{2} u(t-1)+r m(t) f(u(t))=$ $0, t \in \mathbb{T}, u(0)=u(T+1)=0$, where $r \in \mathbb{R}$ is a parameter, $m: \mathbb{T} \rightarrow \mathbb{R}$ changes its sign and $m(t) \neq 0$ for $t \in \mathbb{T}$.

\section{Introduction}

Let $T>5$ be an integer, $\mathbb{T}=\{1,2, \ldots, T\}$. In this paper, we are concerned with the global structure of positive solutions set of the discrete second-order boundary value problems

$$
\begin{gathered}
\Delta^{2} u(t-1)+r m(t) f(u(t))=0, \quad t \in \mathbb{T}, \\
u(0)=u(T+1)=0,
\end{gathered}
$$

where $r \in \mathbb{R}$ is a parameter, $m: \mathbb{T} \rightarrow \mathbb{R}$ changes its sign, and $m(t) \neq 0$ for $t \in \mathbb{T}$.

The boundary value problems with sign-changing weight arise from a selectionmigration model in population genetics, see Fleming [1]. $m$ changes sign corresponds to the fact that an allele $A_{1}$ holds an advantage over a rival allele $A_{2}$ at the same points and is at a disadvantage at others. The parameter $r$ corresponds to the reciprocal of the diffusion. So, the existence and multiplicity of positive solutions with sign-changing weight in continuous case has been studied by many authors, see, for example, [2-8] and the references therein. 
For the discrete case, there are many literature dealing with difference equations similar to (1.1) subject to various boundary value conditions. We refer to [9-17] and the references therein. However, there are few papers to discuss the existence of positive solutions to (1.1) and (1.2) if $m(t)$ changes sign on $\mathbb{T}$. Maybe the main reason is the spectrum of the following linear eigenvalue problems

$$
\begin{gathered}
\Delta^{2} u(t-1)+\lambda m(t) u(t)=0, \quad t \in \mathbb{T}, \\
u(0)=u(T+1)=0
\end{gathered}
$$

is not clear when $m$ changes its sign on $\mathbb{T}$.

In 2008, Shi and Yan [18] investigated the spectrum of the second-order boundary value problems of difference equations

$$
\begin{gathered}
-\Delta[p(t-1) \Delta u(t-1)]+q(t) u(t)=\lambda m(t) u(t), \quad t \in \mathbb{T} \\
\left(\begin{array}{c}
p(0) \Delta u(0) \\
-p(T) \Delta u(T)
\end{array}\right)=K\left(\begin{array}{c}
u(0) \\
u(T)
\end{array}\right)
\end{gathered}
$$

where $K=\left(\begin{array}{ll}k_{11} & k_{12} \\ k_{21} & k_{22}\end{array}\right)$ is a symmetric and positive definite $2 \times 2$ matrix. $p:\{0,1, \ldots, T\} \rightarrow \mathbb{R}$, $q: \mathbb{T} \rightarrow \mathbb{R}$, and $m: \mathbb{T} \rightarrow \mathbb{R}$ satisfy

(A1) $m: \mathbb{T} \rightarrow \mathbb{R}$ changes its sign, and $m(t) \neq 0$ for $t \in \mathbb{T}$.

(A2) $p(t-1) \geq 0, q(t) \geq 0, p(t-1)+q(t)>0$, for $t \in \mathbb{T}$, and $p(0)+k_{11} \neq 0, p(T)>0$.

They proved that (1.4) has $T$ eigenvalues $\lambda_{1} \leq \lambda_{2} \leq \cdots \leq \lambda_{T}$ which have $T$ corresponding linearly independent and orthogonal eigenfunctions.

However, it's easy to see that (1.3) is not included in (1.4) under the condition (A2). Also, Shi and Yan [18] provided no information about the sign of the eigenvalues and no information about the corresponding eigenfunctions.

In this paper, we will show that (1.3) has two principal eigenvalues $\lambda_{m,-}<0<\lambda_{m,+}$, and the corresponding eigenfunctions we denote by $\psi_{m,-}$ and $\psi_{m,+}$ don't change their sign on $\widehat{\mathbb{T}}:=\{0,1, \ldots, T, T+1\}$. Based on this result, using Rabinowitz's global bifurcation theorem [19], we will discuss the global structure of positive solutions set of (1.1) and (1.2).

The assumptions we are interested in this paper are as follows:

(H1) $f \in C(\mathbb{R}, \mathbb{R})$ with $s f(s)>0$ for $s \neq 0$;

(H2) $f_{0}=\lim _{|s| \rightarrow 0}(f(s) / s) \in(0, \infty)$;

(H3) $f_{\infty}=\lim _{|s| \rightarrow+\infty}(f(s) / s)=0$;

(H3') $f_{\infty}=\infty$.

Our main result is the following.

Theorem 1.1. Assume that (H1), (H2) hold.

(i) If (H3) holds, then there exist $0<\lambda^{*} \leq \lambda_{m,+} / f_{0}$ and $\lambda_{m,-} / f_{0} \leq \lambda_{*}<0$ such that (1.1), (1.2) has at least one positive solution for $r \in\left(-\infty, \lambda_{*}\right) \cup\left(\lambda^{*}, \infty\right)$.

(ii) If (H3') holds, then there exist $\rho_{*} \leq \lambda_{m,-} / f_{0}<0$ and $\rho^{*} \geq \lambda_{m,+} / f_{0}>0$ such that (1.1), (1.2) has at least one positive solution for $r \in\left(\rho_{*}, 0\right) \cup\left(0, \rho^{*}\right)$. 
The rest of the paper is arranged as follows. In Section 2, the existence of two principal eigenvalues of (1.3), and some properties of these two eigenvalues will be discussed. In Section 3, we will prove our main result.

\section{Existence of Two Principal Eigenvalues to (1.3)}

Recall that $\mathbb{T}=\{1,2, \ldots, T\}$ and $\widehat{\mathbb{T}}=\{0,1, \ldots, T+1\}$. Let $X=\{u: \widehat{\mathbb{T}} \rightarrow \mathbb{R} \mid u(0)=u(T+1)=0\}$. Then $X$ is a Banach space under the norm $\|u\|_{X}=\max _{t \in \widehat{\mathbb{T}}}|u(t)|$. Let $Y=\{u \mid u: \mathbb{T} \rightarrow \mathbb{R}\}$. Then $Y$ is a Banach space under the norm $\|u\|_{Y}=\max _{t \in \mathbb{T}}|u(t)|$.

It's well known that the operator $X: X \rightarrow Y$,

$$
\chi(0, u(1), u(2), \ldots, u(T), 0)=(u(1), u(2), \ldots, u(T))
$$

is a homomorphism.

Define the operator $L: X \rightarrow Y$ by

$$
L u(t)=-\Delta^{2} u(t-1), \quad t \in \mathbb{T}
$$

In this section, we will discuss the existence of principal eigenvalues for (1.3) with $m: \mathbb{T} \rightarrow \mathbb{R}$ that changes its sign. The main idea we will use aries from $[20,21]$.

Theorem 2.1. Equation (1.3) has two principal eigenvalues $\lambda_{m,+}$ and $\lambda_{m,-}$ such that $\lambda_{m,-}<0<\lambda_{m,+}$, and the corresponding eigenfunctions, we denoted by $\psi_{m,+}$ and $\psi_{m,-}$ do not change sign on $\widehat{\mathbb{T}}$.

Proof. Consider, for fixed $\lambda$, the eigenvalue problems

$$
\begin{gathered}
L u-\lambda m(t) u(t)=\mu u(t), \quad t \in \mathbb{T}, \\
u(0)=u(T+1)=0 .
\end{gathered}
$$

By Kelley and Peterson [22, Theorem 7.6], for fixed $\lambda,(2.3)$ has $T$-simple eigenvalues

$$
\mu_{m, 1}(\lambda)<\mu_{m, 2}(\lambda)<\cdots<\mu_{m, T}(\lambda)
$$

and the corresponding eigenfunction $\psi_{m, k}(\lambda, t)$ has exactly $k-1$ simple generalized zeros.

Thus, $\lambda$ is a principal eigenvalue of (1.3) if and only if $\mu_{m, 1}(\lambda)=0$.

On the other hand, let

$$
S_{m, \lambda}=\left\{\sum_{t=0}^{T}|\Delta \phi(t)|^{2}-\lambda \sum_{t=1}^{T} m(t) \phi(t)^{2}: \phi \in X, \sum_{t=1}^{T} \phi(t)^{2}=1\right\}
$$

Clearly, $S_{m, \lambda}$ is bounded below. Then $\mu_{m, 1}(\lambda)=\inf S_{m, \lambda}$.

For fixed $\phi \in X, \lambda \rightarrow \sum_{t=0}^{T}|\Delta \phi(t)|^{2}-\lambda \sum_{t=1}^{T} m(t) \phi^{2}(t)$ is an affine and so concave function. As the infimum of any collection of concave functions is concave, it follows that $\lambda \rightarrow \mu_{m, 1}(\lambda)$ is a concave function. Also, by considering test functions $\phi_{1}, \phi_{2} \in X$ such that 
$\sum_{t=1}^{T} m(t) \phi_{1}^{2}(t)<0$ and $\sum_{t=1}^{T} m(t) \phi_{2}^{2}(t)>0$, it is easy to see that $\mu_{m, 1}(\lambda) \rightarrow-\infty$ as $\lambda \rightarrow \pm \infty$. Thus, $\lambda \rightarrow \mu_{m, 1}(\lambda)$ is an increasing function until it attains its maximum and is a decreasing function thereafter.

Since $\mu_{1}(0)>0, \lambda \rightarrow \mu_{m, 1}(\lambda)$ must have exactly two zeros. Thus, (1.3) has exactly two principal eigenvalues, $\lambda_{m,+}>0$ and $\lambda_{m,-}<0$, and the corresponding eigenfunctions we denoted by $\psi_{m,+}$ and $\psi_{m,-}$ don't change sign on $\widehat{\mathbb{T}}$.

Remark 2.2. From the proof of Theorem 2.1, it is not difficult to see that the following results hold.

(i) If $m(t) \geq 0$ on $\mathbb{T}$ and there exist at least one point $t_{0} \in \mathbb{T}$ such that $m\left(t_{0}\right)>0$, then (1.3) has only one principal eigenvalue $\lambda_{m,+}>0$.

(ii) If $m(t) \leq 0$ on $\mathbb{T}$ and there exist at least one point $t_{0} \in \mathbb{T}$ such that $m\left(t_{0}\right)<0$, then (1.3) has only one principal eigenvalue $\lambda_{m_{,-}}<0$.

Now, we give some properties for the above principal eigenvalue(s).

Theorem 2.3. Let $m: \mathbb{T} \rightarrow \mathbb{R}$ change its sign. Assume that there exists $m^{\prime}: \mathbb{T} \rightarrow \mathbb{R}$ such that $m(t) \leq m^{\prime}(t)$ for $t \in \mathbb{T}$. Then the followings hold.

(i) If $m^{\prime}$ changes sign on $\mathbb{T}$, then $\lambda_{m^{\prime},-} \leq \lambda_{m,-}, \lambda_{m^{\prime},+} \leq \lambda_{m,+}$;

(ii) If $m^{\prime} \geq 0$, then $0<\lambda_{m^{\prime},+} \leq \lambda_{m,+}$.

Proof. For convenience, we only prove the case (i). It can be seen that for $\lambda>0, S_{m, \lambda} \geq S_{m^{\prime}, \lambda}$, which implies $\mu_{m, 1}(\lambda) \geq \mu_{m^{\prime}, 1}(\lambda)$ and consequently, $\lambda_{m,+} \geq \lambda_{m^{\prime},+}$.

On the other hand, for $\lambda<0, S_{m, \lambda} \leq S_{m^{\prime}, \lambda}$, which implies $\mu_{m, 1}(\lambda) \leq \mu_{m^{\prime}, 1}(\lambda)$ and consequently, $\lambda_{m,-} \geq \lambda_{m^{\prime},-}$.

Suppose that $\mathbb{T}_{0}=\{a+1, a+2, \ldots, b-1\}$ is a strict subset of $\mathbb{T}$, and $m_{\mathbb{T}_{0}}$ denote the restriction of $m$ on $\mathbb{T}_{0}$. Consider the linear eigenvalue problems

$$
\begin{gathered}
\Delta^{2} u(t-1)+\lambda m(t) u(t)=0, \quad t \in \mathbb{T}_{0}, \\
u(a)=u(b)=0 .
\end{gathered}
$$

Then we get the following result.

Theorem 2.4. (i) If $m(t)>0$ for $t \in \mathbb{T}_{0}$, then (2.6) has only one positive principal eigenvalue $\lambda_{m_{\mathbb{T}_{0}},+}$ such that $0<\lambda_{m,+}<\lambda_{m_{\mathbb{T}_{0}},+}$.

(ii) If $m(t)<0$ for $t \in \mathbb{T}_{0}$, then (2.6) has only one principal eigenvalue $\lambda_{m_{\mathbb{T}_{0}},-}$ such that $\lambda_{m_{\mathbb{T}_{0},}}<\lambda_{m_{,-}}$.

(iii) If $m$ changes its sign on $\mathbb{T}_{0}$, then (2.6) has two principal eigenvalue $\lambda_{m_{\mathbb{T}_{0}},-}<0$ and $\lambda_{m_{\mathbb{T}_{0}},+}>0$ such that

$$
\lambda_{m,+}<\lambda_{m_{\mathbb{T}_{0}},+}, \quad \lambda_{m_{\mathbb{T}_{0}},-}<\lambda_{m_{,}-}
$$

Proof. Consider the following problems:

$$
\begin{gathered}
-\Delta^{2} u(t-1)-\lambda m(t) u(t)=\mu u(t), \quad t \in \mathbb{T}_{0}, \\
u(a)=u(b)=0 .
\end{gathered}
$$


Let $\mu_{m_{\mathbb{T}_{0}}, k}$ denote the $k$ th eigenvalue of (2.8), and $\psi_{m_{\mathbb{T}_{0}}, k}$ the corresponding eigenfunction which has exactly $k-1$ generalized zeros in $(a, b)$. Let $E=\{u:\{a, \ldots, b\} \rightarrow \mathbb{R} \mid u(a)=$ $u(b)=0\}$ be a Banach space under the norm $\|u\|_{E}=\max \{|u(t)|: t \in\{a, a+1, \ldots, b-1, b\}\}$. Let

$$
S_{m_{\mathbb{T}_{0}}, \lambda}=\left\{\sum_{t=a}^{b-1}|\Delta \phi(t)|^{2}-\lambda \sum_{t=a+1}^{b-1} m(t) \phi(t)^{2}: \phi \in E, \sum_{t=a+1}^{b-1} \phi(t)^{2}=1\right\} .
$$

We get $\mu_{m_{\mathbb{T}_{0}}, 1}=\inf S_{m_{\mathbb{T}_{0}}, \lambda}$. Similar to the proof of Theorem 2.1, we get the following assertions.

(i) If $m(t)>0$ for $t \in \mathbb{T}_{0}$, then (2.6) has only one principal eigenvalue $\lambda_{m_{\mathbb{T}_{0}}+}>0$.

(ii) If $m(t)<0$ for $t \in \mathbb{T}_{0}$, then (2.6) has only one negative principal eigenvalue $\lambda_{m_{\mathbb{T}_{0}},-}<$ 0 .

(iii) If $m$ changes its sign on $\mathbb{T}_{0}$, then (2.6) has two principal eigenvalue $\lambda_{m_{\mathbb{T}_{0}},-}<0$ and $\lambda_{m_{\mathbb{T}_{0}},+}>0$.

Now, we prove that the inequalities in (i), (ii), and (iii) hold. For convenience, suppose that $\sum_{t=a+1}^{b-1} \psi_{m_{\mathbb{T}_{0}}, 1}^{2}(\lambda, t)=1$.

Let $\widetilde{\psi}_{m_{\mathbb{T}_{0}}, 1}$ denote the extension of $\psi_{m_{\mathbb{T}_{0}}, 1}$ by zero on $\mathbb{T}$, that is,

$$
\widetilde{\psi}_{m_{\mathbb{T}_{0}}, 1}(\lambda, t)= \begin{cases}\psi_{m_{\mathbb{T}_{0}}, 1}(\lambda, t), & t \in \mathbb{T}_{0} \\ 0, & t \in \mathbb{T} \backslash \mathbb{T}_{0}\end{cases}
$$

Then,

$$
\begin{aligned}
\mu_{m_{\mathbb{T}_{0}}, 1}(\lambda) & =\sum_{t=a}^{b-1}\left|\Delta \psi_{m_{\mathbb{T}_{0}}, 1}(\lambda, t)\right|^{2}-\lambda \sum_{t=a+1}^{b-1} m(t) \psi_{m_{\mathbb{T}_{0}}, 1}^{2}(\lambda, t) \\
& =\sum_{t=1}^{T}\left|\Delta \widetilde{\psi}_{m_{\mathbb{T}_{0}}, 1}(\lambda, t)\right|^{2}-\lambda \sum_{t=1}^{T} m(t) \widetilde{\psi}_{m_{\mathbb{T}_{0}},}^{2}(\lambda, t) \\
& >\inf _{v \in X}\left\{\sum_{t=1}^{T}|\Delta v(\lambda, t)|^{2}-\lambda \sum_{t=1}^{T} m(t) v^{2}(\lambda, t), \sum_{t=1}^{T} v^{2}(\lambda, t)=1\right\} \\
& =\mu_{m, 1}(\lambda)
\end{aligned}
$$

which implies the desired results.

\section{The Proof of the Main Result}

First, we deal with the case $r>0$.

Recall that $L: X \rightarrow Y$,

$$
L u(t)=-\Delta^{2} u(t-1)
$$


Let $\zeta, \xi \in C(\mathbb{R}, \mathbb{R})$ be such that

$$
f(u)=f_{0} u+\zeta(u), \quad f(u)=f_{\infty} u+\xi(u) .
$$

Clearly,

$$
\lim _{|u| \rightarrow 0} \frac{\zeta(u)}{u}=0, \quad \lim _{|u| \rightarrow \infty} \frac{\xi(u)}{u}=0 .
$$

Let

$$
\tilde{\xi}(u)=\max _{0 \leq s \leq u}|\xi(s)|
$$

Then, $\tilde{\xi}$ is nondecreasing and

$$
\lim _{|u| \rightarrow \infty} \frac{\tilde{\xi}(u)}{u}=0
$$

Let us consider

$$
L u-\lambda m(t) r f_{0} u-\lambda m(t) r \zeta(u)=0,
$$

as a bifurcation problem from the trivial solution $u \equiv 0$.

Equation (3.6) can be converted to the equivalent equation

$$
u(t)=\lambda L^{-1}\left[m(\cdot) r f_{0} u(\cdot)+m(\cdot) r \zeta(u(\cdot))\right](t) .
$$

Further, we note that $\left\|L^{-1}[m(\cdot) \zeta(u(\cdot))]\right\|=o(\|u\|)$ for $u$ near 0 in $X$, since

$$
\begin{aligned}
\left\|L^{-1}[m(\cdot) \zeta(u(\cdot))]\right\| & =\max _{t \in \mathbb{T}}\left|\sum_{s=1}^{T} G(t, s) m(s) \zeta(u(s))\right| \\
& \leq C \cdot \max _{s \in \mathbb{T}}|m(s)|\|\zeta(u(\cdot))\|,
\end{aligned}
$$

where

$$
G(t, s)=\frac{1}{T+1} \begin{cases}(T+1-t) s, & 0 \leq s \leq t \leq T+1 \\ (T+1-s) t, & 0 \leq t \leq s \leq T+1\end{cases}
$$

The results of Rabinowitz [19] for (3.6) can be stated as follows: from $\left(\lambda_{m,+} / r f_{0}, 0\right)$, there emanates an unbounded continua $\mathcal{C}_{+}$of positive solutions in $\mathbb{R} \times X$.

It is clear that any solution of (3.6) of the form $(1, u)$ yields a solution $u$ of $(1.1)$ and (1.2). So, we focus on the shape of $\mathcal{C}_{+}$under the conditions (H1)-(H3) or (H1)-(H3'), and we will show that $\mathcal{C}_{+}$crosses the hyperplane $\{1\} \times X$ in $\mathbb{R} \times X$. 
Lemma 3.1. Suppose that (H1)-(H3) hold. Let $J=[a, b]$ be a given compact interval in $(0, \infty)$. Then for all $r \in J$, there exists $M_{J}>0$ such that all possible positive solution $u$ of (1.1) and (1.2) satisfy $\|u\|_{X} \leq M_{J}$.

Proof. Suppose on the contrary that there exists a sequence $\left\{y_{n}\right\}$ of positive solutions for (3.6) with $\left\{\mu_{n}\right\} \subset J$ and $\left\|y_{n}\right\|_{X} \rightarrow \infty$. Let $\alpha \in(0,1 / b Q)$, where $Q=\sum_{s=1}^{T} G(s, s)|m(s)|$. Then, by (H3), there exists $u_{\alpha}>0$ such that $u>u_{\alpha}$ implies $f(u)<\alpha u$.

Let $K_{\alpha}=\max _{u \in\left[0, u_{\alpha}\right]} f(u)$ and let $A_{n}=\left\{t \in \widehat{\mathbb{T}} \mid y_{n}(t) \leq u_{\alpha}\right\}$ and $B_{n}=\left\{t \in \widehat{\mathbb{T}} \mid y_{n}(\mathrm{t})>u_{\alpha}\right\}$. Then we have

$$
\begin{aligned}
y_{n}(t) & =\mu_{n} \sum_{s=1}^{T} G(t, s) m(s) f\left(y_{n}(s)\right) \\
& =\mu_{n} \sum_{A_{n}} G(t, s) m(s) f\left(y_{n}(s)\right)+\mu_{n} \sum_{B_{n}} G(t, s) m(s) f\left(y_{n}(s)\right) \\
& \leq \mu_{n} K_{\alpha} Q+\mu_{n} \sum_{B_{n}} G(t, s) m(s) f\left(y_{n}(s)\right)
\end{aligned}
$$

for $t \in \widehat{\mathbb{T}}$. Thus,

$$
\frac{1}{\mu_{n}} \leq \frac{K_{\alpha} Q}{\left\|y_{n}\right\|_{X}}+\sum_{B_{n}} G(t, s) m(s) \frac{f\left(y_{n}(s)\right)}{\left\|y_{n}\right\|_{X}}
$$

On $B_{n}, y_{n}(s)>u_{\alpha}$ implies $f\left(y_{n}(s)\right) /\left\|y_{n}\right\|_{X}<f\left(y_{n}(s)\right) / y_{n}(s)<\alpha$. Thus,

$$
\frac{1}{\mu_{n}} \leq \frac{K_{\alpha} Q}{\left\|y_{n}\right\|_{X}}+\alpha \sum_{B_{n}} G(s, s)|m(s)| \leq \frac{K_{\alpha} Q}{\left\|y_{n}\right\|_{X}}+\alpha Q .
$$

Since $0<a \leq \mu_{n} \leq b$ for all $n$, we have $1 / \mu_{n} \geq 1 / b$ for all $n$, and, thus,

$$
\frac{1}{b} \leq \frac{K_{\alpha} Q}{\left\|y_{n}\right\|_{X}}+\alpha Q
$$

By the fact $\left\|y_{n}\right\|_{X} \rightarrow \infty$ as $n \rightarrow \infty$, we get

$$
\frac{1}{b} \leq \alpha Q<\frac{1}{b}
$$

This contradiction completes the proof.

Lemma 3.2. Suppose that (H1)-(H3) hold. Then $\operatorname{Proj}_{\mathbb{R}} \mathcal{C}_{+} \supset\left[\lambda_{m,+} / r f_{0},+\infty\right)$.

Proof. Assume on the contrary that $\sup \left\{\lambda \mid(\lambda, y) \in \mathcal{C}_{+}\right\}<\infty$, then, there exists a sequence $\left\{\left(\mu_{n}, y_{n}\right)\right\} \in \mathcal{C}_{+}$such that

$$
\lim _{n \rightarrow \infty}\left\|y_{n}\right\|_{X}=\infty, \quad \mu_{n}<C_{0}
$$


for some positive constant $C_{0}$ independent of $n$, since $C_{+}$is unbounded. On the other hand, $\mu_{n}>0$ for all $n \in \mathbb{N}$, since $(0,0)$ is the only solution of (3.6) for $\lambda=0$ and $\mathcal{C}_{+} \cap(\{0\} \times X)=\emptyset$. Meanwhile, $\left\{\left(\mu_{n}, y_{n}\right)\right\}$ satisfy

$$
\begin{gathered}
\Delta^{2} y_{n}(t-1)+\mu_{n} m(t) \frac{f\left(y_{n}(t)\right)}{y_{n}(t)} y_{n}(t)=0, \quad t \in \mathbb{T}, \\
y_{n}(0)=y_{n}(T+1)=0 .
\end{gathered}
$$

By (H3), there exist a positive constant $L_{f}>0$ such that $f(u) \leq L_{f} u$. Define a function $x$ : $\mathbb{T} \rightarrow[0, \infty)$ by

$$
x(t)= \begin{cases}L_{f}, & m(t)>0 \\ 0, & m(t)<0\end{cases}
$$

Then, $m(t)\left(f\left(y_{n}(t)\right) / y_{n}(t)\right) \leq \chi(t) m(t)$. Let $\gamma_{+}$be the principal eigenvalue of linear eigenvalue problem

$$
\begin{gathered}
\Delta^{2} v(t-1)+\gamma X(t) m(t) v(t)=0, \quad t \in \mathbb{T} \\
v(0)=v(T+1)=0 .
\end{gathered}
$$

Then, by Remark 2.2, $\gamma_{+}>0$. Subsequently, by Theorem 2.3, we know that

$$
\gamma_{+} \leq \mu_{n}
$$

This combine with Lemma 3.1, $\lim _{n \rightarrow \infty} \mu_{n}=\infty$, which contradicts (3.15). Thus,

$$
\operatorname{Proj}_{\mathbb{R}} \mathcal{C}_{+} \supset\left[\frac{\lambda_{m,+}}{r f_{0}}, \infty\right)
$$

Now, by Lemma 3.2, $\mathcal{C}_{+}$crosses the hyperplane $\{1\} \times X$ in $\mathbb{R} \times X$, and, then, Theorem 1.1(i) holds. To obtain Theorem 1.1(ii), we need to prove the following Lemma.

Lemma 3.3. Suppose that (H1), (H2), and (H3') hold. Then $\operatorname{Proj}_{\mathbb{R}} \mathcal{C}_{+} \supset\left(0, \lambda_{m,+} / r f_{0}\right)$.

Proof. Let $\left\{\left(\mu_{n}, y_{n}\right)\right\} \subset \mathcal{C}_{+}$be such that $\left|\mu_{n}\right|+\left\|y_{n}\right\|_{X} \rightarrow \infty$ as $n \rightarrow \infty$. Then,

$$
\begin{gathered}
\Delta^{2} y_{n}(t-1)+\mu_{n} m(t) f\left(y_{n}(t)\right)=0, \quad t \in \mathbb{T} \\
y_{n}(0)=y_{n}(T+1)=0 .
\end{gathered}
$$


If $\left\{\left\|y_{n}\right\|\right\}$ is bounded, say, $\left\|y_{n}\right\| \leq M_{1}$, for some $M_{1}$ independent of $n$, then we may assume that

$$
\lim _{n \rightarrow \infty} \mu_{n}=\infty
$$

Note that

$$
\frac{f\left(y_{n}(t)\right)}{y_{n}(t)} \geq \inf \left\{\frac{f(s)}{s} \mid 0<s \leq M_{1}\right\}>0
$$

Then, there exist two constants $M_{3}>0, M_{2}>0$ such that

$$
0<M_{2}<\frac{f\left(y_{n}(t)\right)}{y_{n}(t)}<M_{3}
$$

Define two functions $\chi_{1}, \chi_{2}: \mathbb{T} \rightarrow(0, \infty)$ by

$$
\chi_{1}=\left\{\begin{array}{ll}
M_{2}, & m(t)>0, \\
M_{3}, & m(t)<0 .
\end{array} \quad \chi_{2}= \begin{cases}M_{3}, & m(t)>0, \\
M_{2}, & m(t)<0 .\end{cases}\right.
$$

Let $\eta_{*}, \eta^{*}$ be the positive principal eigenvalue of the linear eigenvalue problems

$$
\begin{gathered}
\Delta^{2} v(t-1)+\eta X_{2}(t) m(t) v(t)=0, \quad t \in \mathbb{T}, \\
v(0)=v(T+1)=0, \\
\Delta^{2} v(t-1)+\eta X_{1}(t) m(t) v(t)=0, \quad t \in \mathbb{T}, \\
v(0)=v(T+1)=0,
\end{gathered}
$$

respectively.

Combining (3.22) and (3.24) with the relation

$$
\begin{gathered}
\Delta^{2} y_{n}(t-1)+r \mu_{n} m(t) \frac{f\left(y_{n}(t)\right)}{y_{n}(t)} y_{n}(t)=0, \quad t \in \mathbb{T}, \\
y_{n}(0)=y_{n}(T+1)=0,
\end{gathered}
$$

using Theorem 2.3, we get

$$
\frac{\eta_{*}}{r} \leq \mu_{n} \leq \frac{\eta^{*}}{r}
$$

This contradicts (3.22). So, $\left\{\left\|y_{n}\right\|_{X}\right\}$ is bounded uniformly for all $n \in \mathbb{N}$.

Now, taking $\left\{\left(\mu_{n}, y_{n}\right)\right\} \subset \mathcal{C}_{+}$be such that

$$
\left\|y_{n}\right\|_{X} \longrightarrow+\infty, \quad \text { as } n \longrightarrow+\infty \text {. }
$$

We show that $\lim _{n \rightarrow \infty} \mu_{n}=0$. 
Suppose on the contrary that, choosing a subsequence and relabeling if necessary, $\mu_{n} \geq$ $b_{0}$ for some constant $b_{0}>0$. By (3.32), there exists $t_{0} \in \mathbb{T}$ such that $y_{n}\left(t_{0}\right)=\left\|y_{n}\right\|_{X}$ and $y_{n}\left(t_{0}\right) \rightarrow+\infty$ as $n \rightarrow+\infty$. Thus,

$$
\mu_{n} \frac{f\left(y_{n}\left(t_{0}\right)\right)}{y_{n}\left(t_{0}\right)} \longrightarrow+\infty, \quad \text { as } n \longrightarrow+\infty
$$

Now, the proof can be divided into two cases.

Case $1\left(m\left(t_{0}\right)>0\right)$. Consider the following linear eigenvalue problems

$$
\begin{gathered}
\Delta^{2} v(t-1)+\alpha m(t) v(t)=0, \quad t=t_{0}, \\
v\left(t_{0}-1\right)=v\left(t_{0}+1\right)=0 .
\end{gathered}
$$

By Theorem 2.4, (3.34) has a positive principal eigenvalue $\alpha_{+}$, and

$$
\mu_{n} \frac{f\left(y_{n}\left(t_{0}\right)\right)}{y_{n}\left(t_{0}\right)} \leq \alpha_{+}
$$

which contradicts (3.33).

Case $2\left(m\left(t_{0}\right)<0\right)$. Since $\left(\mu_{n}, y_{n}\right)$ is a solution of $(3.7)$, we get

$$
0 \geq \Delta^{2} y_{n}\left(t_{0}-1\right)=-\mu_{n} r m\left(t_{0}\right) f\left(y_{n}\left(t_{0}\right)\right)>0 \text {. }
$$

This is a contradiction.

Thus, $\lim _{n \rightarrow \infty} \mu_{n}=0$.

At last, we deal with the case $r<0$.

Let us consider

$$
\operatorname{Lu}-\operatorname{\lambda rm}(t) f_{0} u-\lambda r m(t) \zeta(u)=0
$$

as a bifurcation problem from the trivial solution $u \equiv 0$. The results of Rabinowitz [19] for (3.37) can be stated as follows: from $\left(\lambda_{m,-} /-r f_{0}, 0\right)$, there emanates an unbounded continua $\mathcal{C}_{-}$of positive solutions in $\mathbb{R} \times X$.

It is clear that any solution of (3.37) of the form $(-1, u)$ yields a solution $u$ of $(1.1)$ and (1.2). Now, our proofs focus on the shape of $\mathcal{C}_{-}$. It will be proved that when (H1)-(H3) hold, then $\operatorname{Proj}_{\mathbb{R}} \mathcal{C}_{-} \supset\left(-\infty, \lambda_{m,-} /-r f_{0}\right)$, and when $(\mathrm{H} 1)-\left(\mathrm{H} 3^{\prime}\right)$ hold, Proj $\mathcal{C}_{-} \supset\left(\lambda_{m,-} /-r f_{0}, 0\right)$, that is, $\mathcal{C}_{-}$crosses the hyperplane $\{-1\} \times X$ in $\mathbb{R} \times X$. Since the proof is similar to the case $r>0$, we omit it.

Remark 3.4. As an application of Theorem 1.1, let us consider nonlinear discrete problem with indefinite weight

$$
\begin{aligned}
\Delta^{2} u(t-1)+r \widehat{m}(t) \widehat{f}(u(t)) & =0, \quad t \in \mathbb{T}, \\
u(0)=0, \quad u(3) & =0,
\end{aligned}
$$


where $\mathbb{T}=\{1,2\}, \widehat{m}: \mathbb{T} \rightarrow \mathbb{R}$ with $\widehat{m}(1)=1$ and $\widehat{m}(2)=-1$, and

$$
\widehat{f}(s)= \begin{cases}s, & s \in[-1,1] \\ \frac{2 s}{1+s^{4}}, & s \in(-\infty,-1) \cup(1, \infty) .\end{cases}
$$

Then,

$$
\widehat{f}_{0}=1, \quad \widehat{f}_{\infty}=0 .
$$

To find the principal eigenvalues of linear eigenvalue problem

$$
\begin{gathered}
\Delta^{2} u(t-1)+\lambda \widehat{m}(t) u(t)=0, \quad t \in \mathbb{T}, \\
u(0)=0, \quad u(3)=0,
\end{gathered}
$$

we rewrite (3.41) to the recursive sequence

$$
u(t+1)=[2-\lambda \widehat{m}(t)] u(t)-u(t-1) .
$$

This together with the initial value condition

$$
u(0)=0, \quad u(1)=1
$$

imply that

$$
\begin{gathered}
u(2)=[2-\lambda \widehat{m}(1)] u(1)-u(0)=2-\lambda, \\
u(3)=[2-\lambda \widehat{m}(2)] u(2)-u(1)=3-\lambda^{2} .
\end{gathered}
$$

The last equation together with the boundary value condition $u(3)=0$ imply that

$$
\lambda_{\hat{m}_{,-}}=-\sqrt{3}, \quad \lambda_{\widehat{m}_{,+}}=\sqrt{3} .
$$

Thus by Theorem 1.1(i), (3.38), has a positive solution if $r \in(-\infty,-\sqrt{3}) \cup(\sqrt{3}, \infty)$.

\section{Acknowledgments}

The authors are grateful to the anonymous referee for their valuable suggestions. R. Ma is supported by NSFC (11061030).

\section{References}

[1] W. H. Fleming, "A selection-migration model in population genetics," Journal of Mathematical Biology, vol. 2, no. 3, pp. 219-233, 1975. 
[2] M. Delgado and A. Suárez, "On the existence and multiplicity of positive solutions for some indefinite nonlinear eigenvalue problem," Proceedings of the American Mathematical Society, vol. 132, no. 6, pp. 1721-1728, 2004.

[3] K. J. Brown and P. Hess, "Stability and uniqueness of positive solutions for a semi-linear elliptic boundary value problem," Differential and Integral Equations, vol. 3, no. 2, pp. 201-207, 1990.

[4] G. A. Afrouzi and K. J. Brown, "Positive mountain pass solutions for a semilinear elliptic equation with a sign-changing weight function," Nonlinear Analysis, vol. 64, no. 3, pp. 409-416, 2006.

[5] K. J. Brown, "Local and global bifurcation results for a semilinear boundary value problem," Journal of Differential Equations, vol. 239, no. 2, pp. 296-310, 2007.

[6] B. Ko and K. J. Brown, "The existence of positive solutions for a class of indefinite weight semilinear elliptic boundary value problems," Nonlinear Analysis, vol. 39, no. 5, pp. 587-597, 2000.

[7] R. Ma and X. Han, "Existence and multiplicity of positive solutions of a nonlinear eigenvalue problem with indefinite weight function," Applied Mathematics and Computation, vol. 215, no. 3, pp. 1077-1083, 2009.

[8] R. Ma and X. Jia, "Global bifurcation of positive solutions of a second-order periodic boundary value problems with indefinite weight," Nonlinear Analysis, vol. 71, no. 5-6, pp. 2119-2125, 2009.

[9] R. P. Agarwal and J. Henderson, "Positive solutions and nonlinear eigenvalue problems for thirdorder difference equations," Computers \& Mathematics with Applications, vol. 36, no. 10-12, pp. 347$355,1998$.

[10] R. P. Agarwal and D. O’Regan, "Boundary value problems for discrete equations," Applied Mathematics Letters, vol. 10, no. 4, pp. 83-89, 1997.

[11] R. P. Agarwal and F. H. Wong, "Existence of positive solutions for nonpositive difference equations," Mathematical and Computer Modelling, vol. 26, no. 7, pp. 77-85, 1997.

[12] I. Rachunkova and C. C. Tisdell, "Existence of non-spurious solutions to discrete Dirichlet problems with lower and upper solutions," Nonlinear Analysis, vol. 67, no. 4, pp. 1236-1245, 2007.

[13] J. Rodriguez, "Nonlinear discrete Sturm-Liouville problems," Journal of Mathematical Analysis and Applications, vol. 308, no. 1, pp. 380-391, 2005.

[14] G. Zhang and S. S. Cheng, "Positive periodic solutions for discrete population models," Nonlinear Functional Analysis and Applications, vol. 8, no. 3, pp. 335-344, 2003.

[15] G. Zhang and S. S. Cheng, "Positive periodic solutions of nonautonomous functional differential equations depending on a parameter," Abstract and Applied Analysis, vol. 7, no. 5, pp. 279-286, 2002.

[16] G. Zhang and W. Feng, "On the number of positive solutions of a nonlinear algebraic system," Linear Algebra and its Applications, vol. 422, no. 2-3, pp. 404-421, 2007.

[17] R. Ma, Y. Xu, and C. Gao, "A global description of the positive solutions of sublinear second-order discrete boundary value problems," Advances in Difference Equations, vol. 2009, Article ID 671625, 15 pages, 2009.

[18] G. Shi and R. Yan, "Spectral theory of left definite difference operators," Journal of Mathematical Analysis and Applications, vol. 337, no. 1, pp. 116-122, 2008.

[19] P. H. Rabinowitz, "Some global results for nonlinear eigenvalue problems," Journal of Functional Analysis, vol. 7, pp. 487-513, 1971.

[20] P. Hess and T. Kato, "On some linear and nonlinear eigenvalue problems with an indefinite weight function," Communications in Partial Differential Equations, vol. 5, no. 10, pp. 999-1030, 1980.

[21] G. A. Afrouzi and K. J. Brown, "On principal eigenvalues for boundary value problems with indefinite weight and Robin boundary conditions," Proceedings of the American Mathematical Society, vol. 127, no. 1, pp. 125-130, 1999.

[22] W. Kelley and A. Peterson, Difference Equations. An Introduction with Applications, Academic Press, New York, NY, USA, Second edition, 2001. 


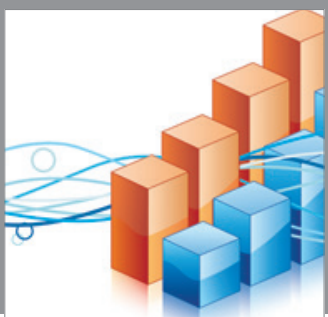

Advances in

Operations Research

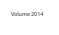

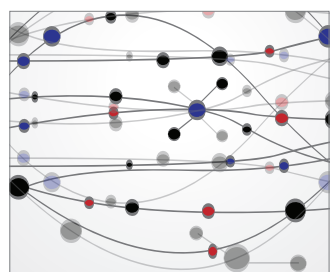

\section{The Scientific} World Journal
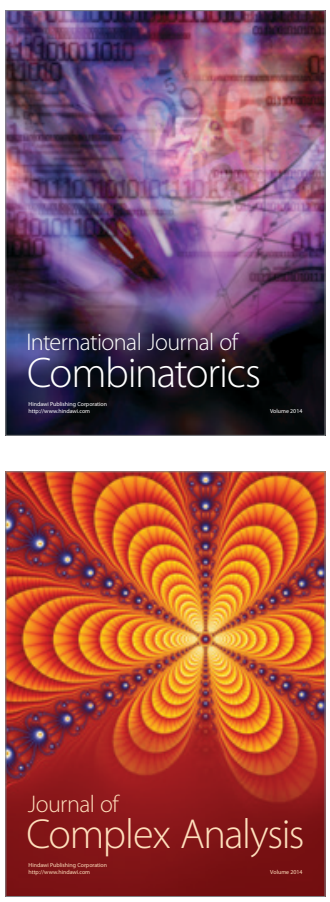

International Journal of

Mathematics and

Mathematical

Sciences
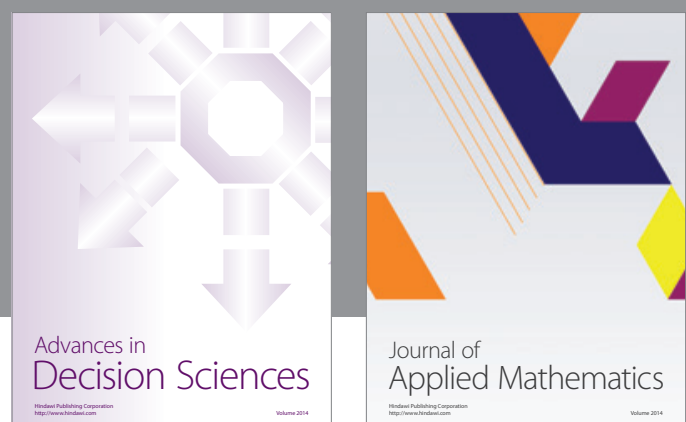

Journal of

Applied Mathematics
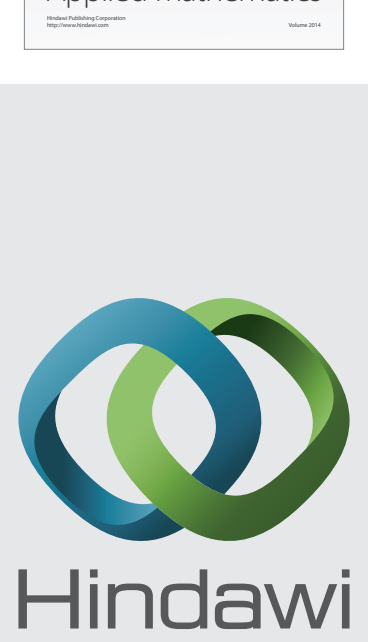

Submit your manuscripts at http://www.hindawi.com
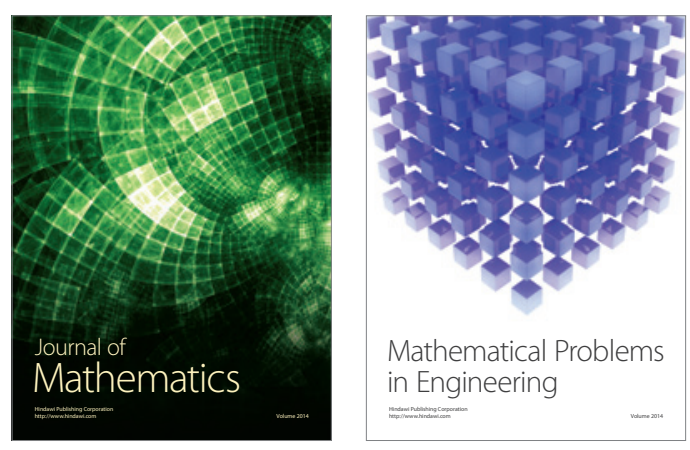

Mathematical Problems in Engineering
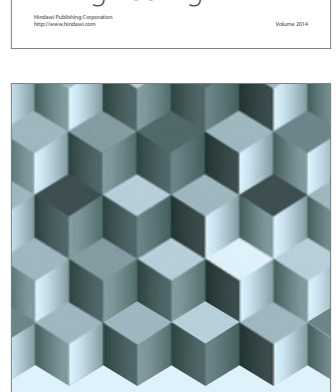

Journal of

Function Spaces
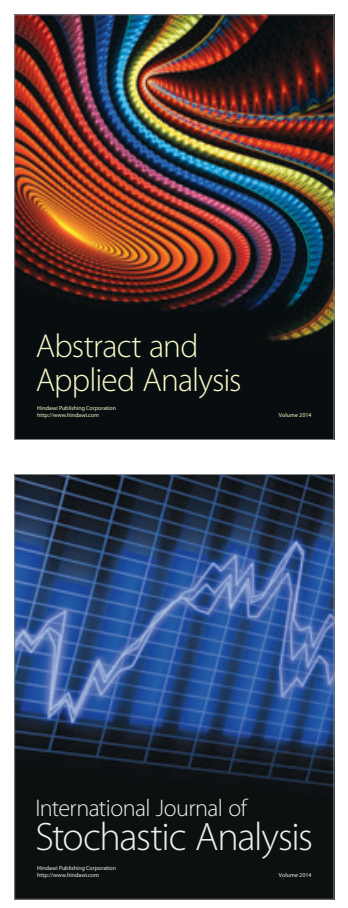

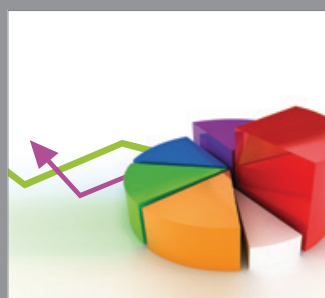

ournal of

Probability and Statistics

Promensencen
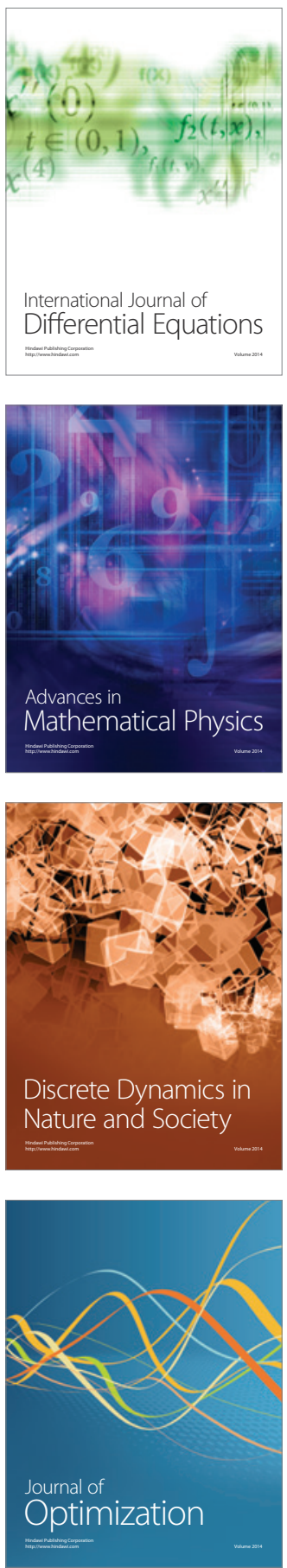